\title{
The TAMORA algorithm: satellite rainfall estimates over West Africa using multi-spectral SEVIRI data
}

\author{
R. S. Chadwick ${ }^{1}$, D. I. F. Grimes ${ }^{1}$, R. W. Saunders ${ }^{2}$, P. N. Francis ${ }^{2}$, and T. A. Blackmore ${ }^{2}$ \\ ${ }^{1}$ Department of Meteorology, University of Reading, Reading, UK \\ ${ }^{2}$ Met Office, Exeter, UK \\ Received: 14 October 2009 - Revised: 16 February 2010 - Accepted: 17 February 2010 - Published: 8 March 2010
}

\begin{abstract}
A multi-spectral rainfall estimation algorithm has been developed for the Sahel region of West Africa with the purpose of producing accumulated rainfall estimates for drought monitoring and food security. Radar data were used to calibrate multi-channel SEVIRI data from MSG, and a probability of rainfall at several different rain-rates was established for each combination of SEVIRI radiances. Radar calibrations from both Europe (the SatPrecip algorithm) and Niger (TAMORA algorithm) were used. 10 day estimates were accumulated from SatPrecip and TAMORA and compared with kriged gauge data and TAMSAT satellite rainfall estimates over West Africa. SatPrecip was found to produce large overestimates for the region, probably because of its non-local calibration. TAMORA was negatively biased for areas of West Africa with relatively high rainfall, but its skill was comparable to TAMSAT for the low-rainfall region climatologically similar to its calibration area around Niamey. These results confirm the high importance of local calibration for satellite-derived rainfall estimates. As TAMORA shows no improvement in skill over TAMSAT for dekadal estimates, the extra cloud-microphysical information provided by multi-spectral data may not be useful in determining rainfall accumulations at a ten day timescale. Work is ongoing to determine whether it shows improved accuracy at shorter timescales.
\end{abstract}

\section{Introduction}

Rainfall estimation over the African continent is subject to multiple challenges, several of which are distinct to the region. Rain-gauge networks are often sparse and unreliable, and precipitation radars are rare. Connected to this lack of observational data, and compounding the problem, is the rel-

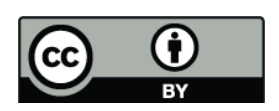

Correspondence to: R. S. Chadwick (r.s.chadwick@rdg.ac.uk) ative inaccuracy of quantitative model rainfall outputs over much of Africa (Diro et al., 2009).

However as the majority of Sub-Saharan Africa is dependent on rain-fed agriculture, near-real-time rainfall estimates are extremely important for agricultural and food security applications. Satellite-based rainfall estimates have been seen for some time as a way to provide rainfall data at the required temporal and spatial scales (Kidd, 2001).

Several such techniques have been designed for rainfall estimation over Africa, including the TAMSAT (Grimes et al., 1999), MIRA (Todd et al., 2001) and RFE (Laws et al., 2004) algorithms. With the exception of TAMSAT, which uses only MSG (Meteosat Second Generation), most such algorithms make use of multiple satellites or instruments. However, as many African Met services are equipped to receive MSG data, it can be argued that a rainfall algorithm using only MSG is more suitable for use in Africa, as it can be more easily adapted or calibrated locally by Met services in the region.

The TAMSAT algorithm uses only a single IR channel from the SEVIRI (Spinning Enhanced Visible and Infrared Imager) instrument on board MSG, but recently much work has been done on utilising multiple channel data from SEVIRI and other similar instruments for cloud classification and rainfall identification.

During the daytime, the $0.8 \mu \mathrm{m}, 1.6 \mu \mathrm{m}$ and $3.9 \mu \mathrm{m}$ channels provide information on cloud optical depth and the cloud microphysical properties of particle effective radius and phase, which in turn provide indirect information about cloud rain-rate. (Thies et al., 2008a; Key and Intrieri, 2000). At night, a combination of IR channels can provide similar information, though with less accuracy than the daytime channels (Thies et al., 2008b; Lensky and Rosenfeld, 2003).

The aim of this project is to determine whether an improved rainfall estimation algorithm for Africa can be devised using the full range of MSG channels. The starting point is the UK Met Office SatPrecip algorithm which uses a

Published by Copernicus Publications on behalf of the European Geosciences Union. 
real-time radar calibration to produce rainfall estimates over Europe. This algorithm, reconfigured and recalibrated to take account of West-African conditions, is referred to from now on as TAMORA (TAMSAT, Met Office Rainfall for Africa).

\section{Data}

\subsection{Selection of SEVIRI channels}

The SEVIRI instrument has 12 channels ranging between $0.635 \mu \mathrm{m}$ and $13.4 \mu \mathrm{m}$ (Morgan, 2005). Only those channels considered most useful (for identification of raining clouds) were selected for use in the TAMORA algorithm.

In preparation for potential rainfall identification applications of multi-spectral SEVIRI data, Capacci and Conway (2005) examined in detail the combinations of MODIS (Moderate resolution Imaging Spectroradiometer) channels which were most useful in identifying raining clouds.

Based on this work, the most useful combination of SEVIRI channels for rainfall identification was taken to be the $0.8,1.6,3.9$ and $10.8 \mu \mathrm{m}$ channels during daytime, and the $3.9,10.8$ and $12.0 \mu \mathrm{m}$ channels at night. These are the combinations used in the UK Met Office SatPrecip and TAMORA algorithms.

\subsection{Radar rain-rate data from Niamey, Niger}

A mobile C-band Doppler radar was operated from Niamey airport (Niger) from 5 July to 27 September 2006 as part of the AMMA (African Monsoon Multidisciplinary Analysis) project. The data for this work were obtained as values of reflectivity in gridded Cartesian format. The gridded data have a $1 \mathrm{~km}$ horizontal and vertical resolution over a $300 \mathrm{~km}$ by $300 \mathrm{~km}$ horizontal area with 19 vertical levels and a $10 \mathrm{~min}$ time resolution.

Data from two tipping-bucket rain-gauges within the area of radar coverage were available, but this was not considered to be a large enough dataset (particularly in terms of number of gauges) to attempt to determine a new $Z-R$ relation using a linear regression of radar reflectivity against gauge data.

Several previous studies (Ochou et al., 2007; Nzeukou et al., 2004) have compared radar reflectivity data with dropsize distribution estimates from disdrometer data to produce Z-R relations for West Africa. After discussion with AMMA scientists working in this area, it was decided to use the $Z-R$ relation of $Z=400 R^{1.3}$ (Gosset, personal communication, 2008). These coefficients fall in the middle of the range of values found in the literature.

After comparison of the radar rain-rate estimates with the two previously mentioned tipping-bucket rain-gauges, the radar was found to be systematically underestimating rainfall. A $+3 \mathrm{dBZ}$ bias correction was applied to the radar reflectivity data before re-converting them to rain-rate, and these corrected estimates were found to agree much more closely with the gauge values.
Table 1. Radar rain-rate (RR) bins used for SatPrecip and TAMORA calibration, and corresponding values of SatPrecip and TAMORA output rain-rate (all $\mathrm{mm} / \mathrm{hr}$ ). Only RR bins $1-4$ are used by SatPrecip over Europe, and only RR bins 1-6 are used by TAMORA.

\begin{tabular}{lccc}
\hline Rain-rate bin & Radar RR range & SatPrecip & TAMORA \\
\hline 1 & $>0-0.125$ & 0 & 0.08 \\
2 & $0.125-0.5$ & 0.125 & 0.44 \\
3 & $0.5-2$ & 0.5 & 1.23 \\
4 & $2.0-4.0$ & 2.0 & 2.71 \\
5 & $4.0-8.0$ & 4.0 & 4.88 \\
6 & $8.0-16.0$ & 8.0 & 11.12 \\
7 & $16.0-32.0$ & 16.0 & NA \\
8 & $32.0-64.0$ & 32.0 & NA \\
9 & $64.0-128.0$ & 64.0 & NA \\
10 & $>128.0$ & 128.0 & NA \\
\hline
\end{tabular}

\subsection{Kriged rain-gauge data from the Sahel}

Rain-gauge data from the Sahel region of West Africa for the rainy season of July - September 2004 were used for validation of satellite rainfall estimates at 0.5 by $0.5^{\circ}$ spatial aggregation, and dekadal (10 day) time scale. The amount of gauge data available varied between dekads, with a minimum of 350 and a maximum of 515 gauges over this season.

The data were interpolated to $0.5^{\circ}$ by $0.5^{\circ}$ scale by regression kriging, as described in Ali et al. (2005). In order to minimise the error in the reference data, only those grid squares which contained at least one gauge were used for comparison with the algorithms.

\section{The SatPrecip algorithm}

The SatPrecip algorithm uses multi-spectral data from SEVIRI to provide instantaneous near-real-time estimates of precipitation over Europe and the North Atlantic. The SEVIRI estimates are calibrated against precipitation radar data from the UK and European networks (Francis et al., 2006).

The radar rain-rate files are first reprojected to MSG resolution, and each MSG image is associated with the radar file that coincides most closely with it temporally. The nonzero radar rain-rates are separated into four classes, shown as rain-rate bins 1-4 in Table 1, and the calibration process is carried out separately for each rain-rate in turn. Only four classes are used operationally over Europe as the product's main purpose is as a qualitative tool for forecasters.

The data from each SEVIRI channel are binned according to brightness temperature (BT) or reflectance (refl.) into a number of discrete classes (32 for each BT channel and 16 for each refl. channel). Combining these multi-channel binned data into a contingency table, a number of satellite pixel data classes are obtained. Taking the radar data as 
"truth", coincident radar and satellite pixels are compared and a probability of rainfall for each satellite data class is computed. This process is performed for each rain-rate threshold used by the algorithm, and in this case 'probability of rainfall' is the probability of a rain-rate greater than the rain-rate threshold.

A threshold probability is then chosen for each rain-rate class, with all satellite data classes above this probability assigned as "raining" (within the rain-rate bin in question), and all others assigned as "not raining" (at this particular rainrate).

The probability threshold is chosen for each rain-rate by the "minimum percent" method (Lovejoy and Austin, 1979), whereby the difference between the number of observed and diagnosed rainy pixels is minimised by an iterative procedure. Cheng and Brown (1995) found that this method of threshold determination produced the most skilful results for bi-spectral instantaneous precipitation estimates.

Having established a rain-rate calibration for the MSG data, the rain-rate estimates can now be extended beyond the area of radar coverage to any region covered by MSG. Validation against UK radar data has shown that the extra SEVIRI channels provide an increase in skill from the original bi-spectral product (Francis et al., 2006).

SatPrecip estimates were initially extended to Africa with only an adjustment to the rain-rate bins. As it was accumulated rainfall that was of interest over Africa, and high rainrates are considerably more common over Africa than over Europe, the number of rain-rate bins was increased to 10 (see Table 1). The SatPrecip output corresponding to each radar rain-rate bin was taken to be the lower threshold of the bin. SatPrecip estimates were computed every hour for July-Sept 2004, and accumulated to dekadal estimates for comparison with rain-gauge data.

\section{The TAMORA algorithm: Adaptation of SatPrecip to Africa}

As the nature of rainfall over Europe (a mixture of stratiform and convective) is very different to that in West Africa (dominated by deep convection), an algorithm (TAMORA) calibrated with precipitation radar data from Africa was considered likely to produce more accurate results over the continent than the original SatPrecip algorithm.

The radar data from Niamey, Niger for JAS 2006 described in Sect. 2.2 were used for the calibration. The data were interpolated to MSG pixel scale, then TAMORA was calibrated by the same method as used for SatPrecip.

As well as using different calibration data, several other changes were made in order to optimise TAMORA for use in Africa. The relationship between SEVIRI brightness temperature/reflectance and probability of rainfall $(\mathrm{P}(\mathrm{R}))$ was found to vary greatly between the European and Niamey data. The binning of radiances in the contingency tables was altered
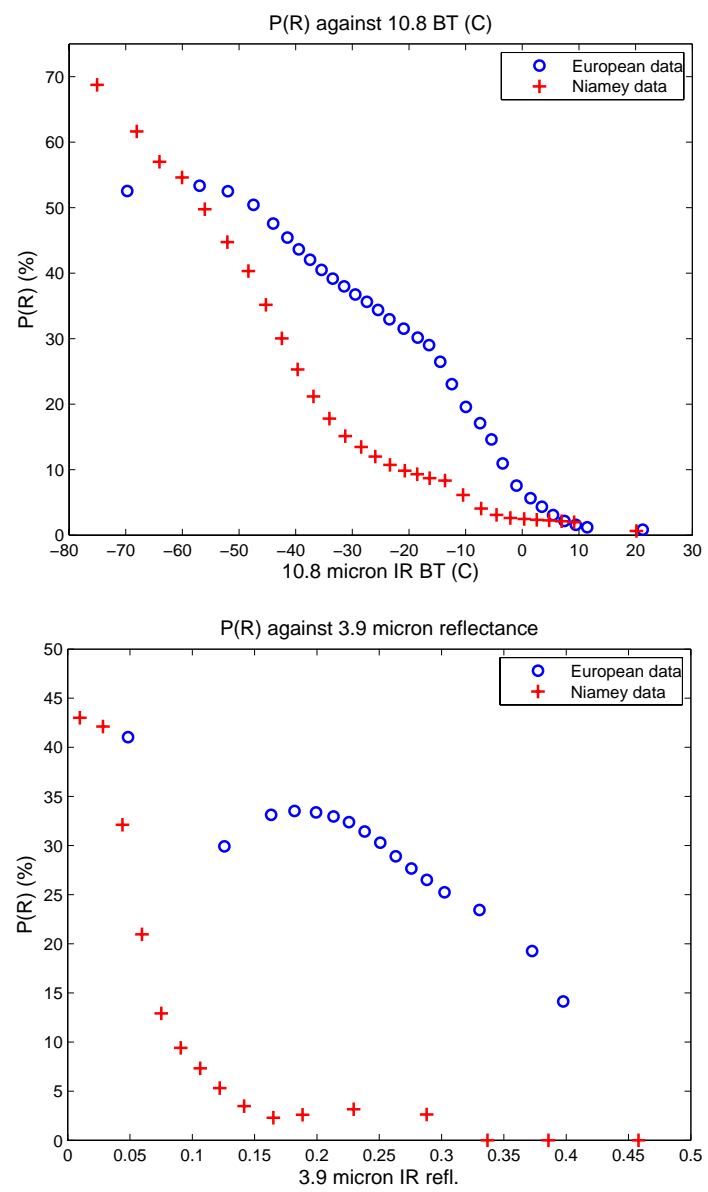

Fig. 1. Probability of rainfall $(\mathrm{P}(\mathrm{R}))$ for radar against nearest SEVIRI pixel for $10.8 \mu \mathrm{m}$ BT (top) and $3.9 \mu \mathrm{m}$ refl. (bottom) from European and Niamey data are shown. Each data point corresponds to one radiance bin in the SatPrecip and TAMORA algorithms respectively. For the $3.9 \mu \mathrm{m}$ channel, only data where the corresponding $10.8 \mu \mathrm{m} \mathrm{BT}$ is $\leq-20^{\circ} \mathrm{C}$ are used in order to eliminate clear sky radiances. European data are for all months combined, Niamey data are for JAS only.

to reflect this, with bin resolution increased for areas where $\mathrm{P}(\mathrm{R})$ was relatively high for Niamey.

Figure 1 shows examples of this change in binning for the $10.8 \mu \mathrm{m}$ BT and $3.9 \mu \mathrm{m}$ refl. channels. For the $10.8 \mu \mathrm{m}$ channel it can be seen that higher bin resolution was required for cold brightness temperatures. This is due to stratiform rain in Europe often occurring from clouds with relatively warm cloud-top temperatures, whereas convective rainfall in $\mathrm{Ni}$ amey is usually only initiated when the cloud top is very high and cold.

For the $3.9 \mu \mathrm{m}$ channel higher resolution was required for very low reflectances. This is due to the differing microphysical properties of European and West African precipitating clouds. European clouds often produce rainfall from water or mixed phase clouds which have a relatively high reflectance 


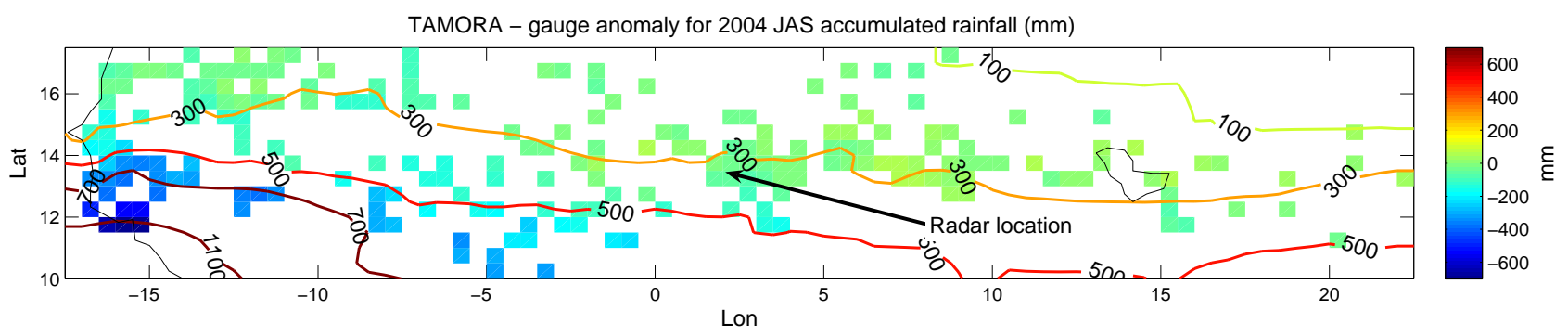

Fig. 2. TAMORA - Kriged Gauge anomaly for accumulated JAS 2004 rainfall (shown by coloured pixels). Only pixels containing at least one gauge are shown. Contours show accumulated JAS 2004 rainfall from kriged gauges (mm). West African coastline and Lake Chad are depicted by black lines, and location of AMMA radar in 2006 is also shown.

Table 2. Summary statistics for evaluation of TAMORA, SatPrecip and TAMSAT against kriged gauge data over the Sahel at $0.5^{\circ}$, 10 day scale. Reduced region estimates are only evaluated over the Sahel sub-region where 2004 JAS gauge rainfall is $\leq 500 \mathrm{~mm}$.

\begin{tabular}{lrcc}
\hline & Bias & RMSE & $R^{2}$ \\
\hline TAMORA & -10.8 & 27.1 & 0.68 \\
SatPrecip & 19.0 & 47.7 & 0.67 \\
TAMSAT & 4.3 & 21.7 & 0.80 \\
TAMORA reduced region & -3.6 & 16.9 & 0.78 \\
SatPrecip reduced region & 21.9 & 46.3 & 0.69 \\
TAMSAT reduced region & 4.4 & 16.0 & 0.79 \\
\hline
\end{tabular}

in the $3.9 \mu \mathrm{m}$ band. Precipitating clouds in Niamey normally contain large ice particles at the top of the cloud, and these correspond to extremely low $3.9 \mu \mathrm{m}$ reflectances. A similar difference between Europe and Niamey was also seen for the $1.6 \mu \mathrm{m}$ channel (not shown here).

The other major change between SatPrecip and TAMORA is the method by which instantaneous estimates are aggregated to longer timescales. SatPrecip simply estimated instantaneous rain-rate every hour and accumulated this directly to longer timescales. However the distribution of instantaneous rain-rates from radar is completely different to an hourly accumulated rainfall distribution, with many more extreme high values at instantaneous scale.

TAMORA estimates were accumulated from half-hourly instantaneous estimates to dekadal accumulations by a more sophisticated method. Radar estimates from the Niamey dataset were averaged over 4 scans (each separated by $10 \mathrm{~min}$ ) in order to produce an estimate of the distribution of half-hourly mean rainfall. Instantaneous rain-rates were then mapped to the equivalent half-hourly mean by histogram matching. The equivalent half-hourly rain-rate (see Table 1) was then used as the output for TAMORA, and accumulated to dekadal timescale. Only rain-rates up to bin 6 were used, as not enough Niamey radar data existed at higher rain-rates for a stable calibration.

\section{Evaluation over West Africa}

An evaluation of SatPrecip, TAMORA and TAMSAT estimates against kriged gauge estimates at dekadal, $0.5^{\circ}$ scale was performed for the period July-September 2004 for the region 10 to $17^{\circ} \mathrm{N},-17.5$ to $21.5^{\circ} \mathrm{E}$. TAMSAT has previously been shown to produce accurate estimates for this region when compared to other satellite rainfall estimates (Jobard et al., 2007). Scatter plots of this validation are shown in Fig. 3, and summary statistics in Table 2.

SatPrecip performs poorly, with a large positive bias and extremely large spread. TAMORA exhibits a large negative bias and large RMSE, mainly due to a tendency to underestimate high rainfall values. Figure 2 shows the TAMORA gauge anomaly for accumulated rainfall JAS 2004, together with contours of rainfall as given by the kriged gauge estimates. It can be seen that TAMORA is largely unbiased for areas of relatively low rainfall (JAS total $\leq 500 \mathrm{~mm}$ ), but displays a negative bias in areas of higher rainfall. TAMSAT performs the best of the three algorithms for the whole validation region.

In order to assess the performance of TAMORA over a region more homogeneously similar to its calibration dataset (around Niamey), the evaluation was repeated for the subset of the validation region where 2004 JAS gauge rainfall is $\leq 500 \mathrm{~mm}$. Results of this are shown in Table 2 and Fig. 3 . SatPrecip continues to perform poorly for this sub-region, but the results for TAMORA are much improved, and its accuracy is comparable to TAMSAT in this case.

\section{Conclusions}

SatPrecip was found to perform poorly over the Sahel region. This can be attributed most obviously to its calibration over Europe. As can be seen from Fig. 1, the relationship between SEVIRI radiances and $\mathrm{P}(\mathrm{R})$ varies greatly between Europe and Africa due to differences in typical rain-producing clouds between the continents. Because of this, SatPrecip will often assign rainfall to relatively low clouds over Africa 

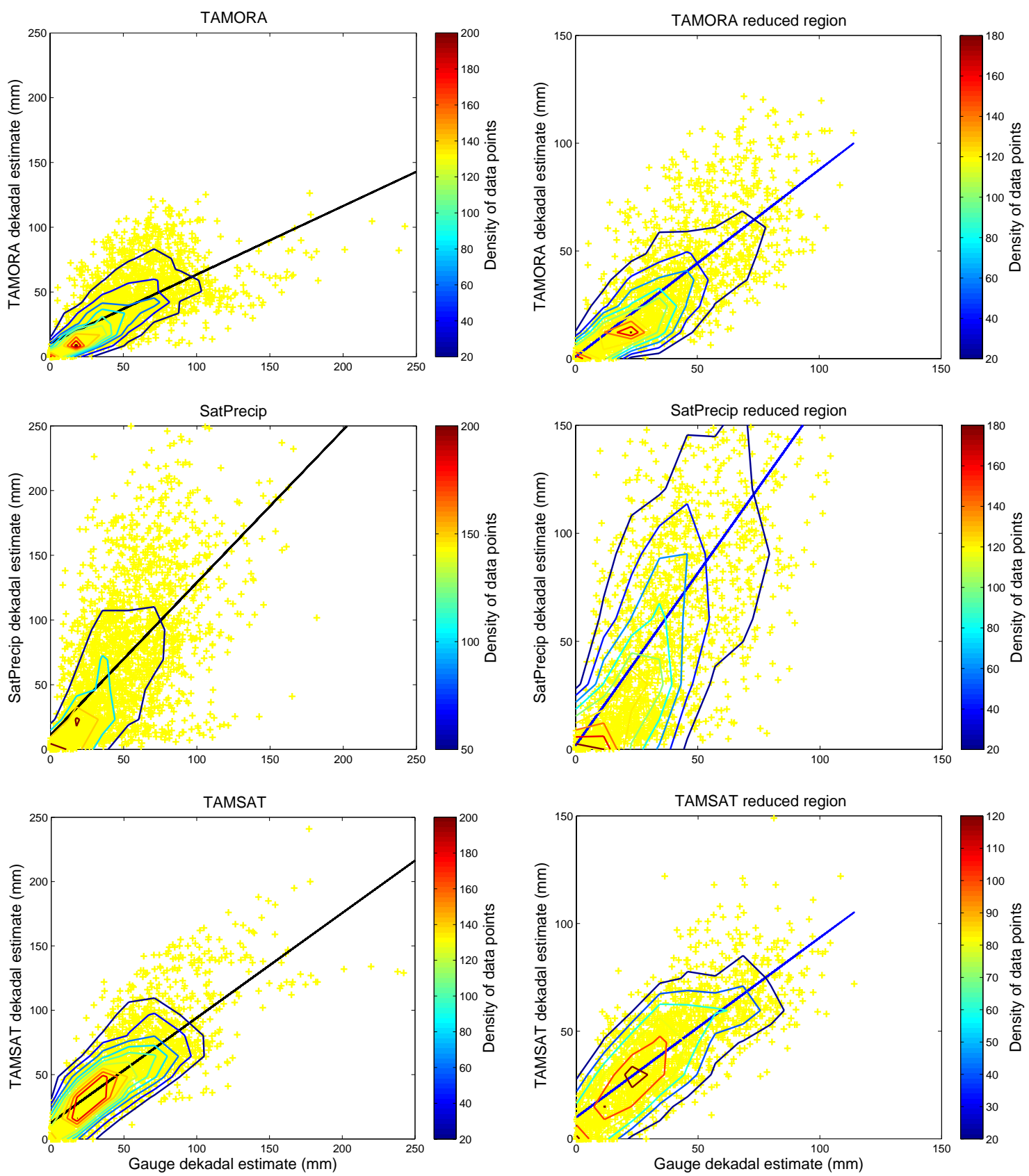

Fig. 3. Density of TAMORA, SatPrecip and TAMSAT estimates plotted against corresponding kriged gauge estimates at $0.5^{\circ}, 10$ day scale, for JAS 2004. Least squares fit lines are also shown. (L) All grid squares within the validation area of 10 to $17^{\circ} \mathrm{N},-17.5$ to $21.5^{\circ} \mathrm{E}$ were used. (R) Only grid squares where the JAS 2004 kriged gauge total was $\leq 500 \mathrm{~mm}$ were used. 
that are in fact unlikely to be raining, and this would explain the large overestimates produced by SatPrecip in the region.

For the Sahel validation region as a whole, TAMORA shows a large negative bias. One possible explanation is that TAMORA is unable to correctly estimate heavy rainfall, as the highest half-hourly rainfall accumulation it can produce is $11.12 \mathrm{~mm}$ (see Table 1). However, if this were the case, then TAMORA would be expected to underestimate rainfall everywhere in the region, because the majority of Sahelian rainfall occurs in short, intense storms. As can be seen in Fig. 2, this is not the case, and for the region where total JAS rainfall is $\leq 500 \mathrm{~mm}$ TAMORA has only a small negative bias.

It appears that TAMORA is only biased for areas of relatively high seasonal precipitation, and in particular those areas which receive more rainfall than its region of calibration in Niamey. This is likely to be the case for a number of reasons. The Guinea region of coastal West Africa has a climate distinct from that of the Sahel, with significant amounts of rain coming from "warm", relatively low-topped clouds. This would cause the opposite problem from that experienced by SatPrecip over the Sahel, with TAMORA unable to correctly identify this rainfall due to its Niamey calibration.

For other regions of TAMORA underestimation, the climate is broadly the same as in Niamey with the majority of rainfall coming from large organised convective systems. However the availability of moisture is greater further south due to the greater proximity of the ocean, and this is likely to be the cause of the underestimation.

Although TAMORA receives information about cloud-top properties from multi-spectral data, this is still very much indirect information about rainfall quantity. Two convective systems with very similar SEVIRI radiance signatures, but in regions of differing moisture availability, may produce quite different accumulated rainfall at the surface. This would suggest that different calibrations of TAMORA may be needed, even between regions of apparently similar climate.

In general, it appears that local calibration for this type of multi-spectral rainfall estimation algorithm is crucial, certainly between continents and regions (as shown by the poor performance of SatPrecip in the Sahel), and even within regions (as seen by the varying performance of TAMORA across the Sahel).

For the reduced, more arid validation region TAMORA's performance for dekadal totals is similar to that of TAMSAT. As TAMSAT uses only a single channel of IR data from SEVIRI it might be expected that TAMORA would perform better than TAMSAT, and there are several possible explanations of why this is not the case.

TAMSAT uses historical rain-gauge data from a long timeseries for calibration, and is calibrated regionally over relatively small, climatologically homogeneous areas, whereas TAMORA only uses one season of radar data from a single radar for calibration. The fact that TAMSAT is calibrated with gauges, not radar means that it is not subject to radar errors (gauge errors over ten days can be considered to be comparatively small). As TAMSAT is calibrated over the same dekadal timescale used here for validation its estimates are better constrained than TAMORA's, with no possibility of unrealistically high values.

Although the extra information about cloud properties available to TAMORA might be expected to compensate for this, it is unclear how useful cloud microphysical data are when estimating convective rainfall at relatively long timescales. While cloud microphysics are important in determining instantaneous convective rain-rates, over larger timescales the accumulated rainfall may simply be proportional to the amount of moisture advected into the upper troposphere. In this case, the multi-channel TAMORA approach would not be expected to produce more skilful estimates than the simple TAMSAT approach over relatively long time periods.

Future work will involve a validation of TAMORA at shorter 1 day and instantaneous timescales to determine if multi-spectral data can improve rainfall estimates at these shorter timescales.

Acknowledgements. The authors would like to thank EUMETSAT for providing the SEVIRI data, AGRHYMET for providing the West African rainfall data, and Earle Williams (MIT) and Nick Guy (San Jose State University) for giving access to the Niamey radar dataset.

Edited by: S. C. Michaelides

Reviewed by: two anonymous referees

\section{References}

Ali, A., Amani, A., and Lebel, T.: Rainfall estimation in the Sahel, Part 1: Error Function, J. Appl. Meteorol., 44, 1691-1706, 2005.

Capacci, D. and Conway, B.: Delineation of precipitation areas from MODIS visible and infrared imagery with artificial neural networks, Meteorol. Appl., 12, 291-305, 2005.

Cheng, A. and Brown, R.: Delineation of Precipitation Areas by Correlation of Meteosat Visible and Infrared Data with radar data, Mon. Weather Rev., 123, 2743-2757, 1995.

Diro, G., Grimes, D., Black, E., O’Neill, A., and Pardo-Iguzquiza, E.: Evaluation of reanalysis rainfall estimates over Ethiopia, Int. J. of Rem. Sens., 29, 67-78, 2009.

Francis, P., Capacci, D., and Saunders, R.: Improving the Nimrod Nowcasting system's satellite precipitation estimates by introducing the new SEVIRI channels, in: EUMETSAT conference proceedings, Helsinki, 12-16 June 2006, 2006.

Grimes, D., Pardo-Iguzquiza, E., and Bonifacio, R.: Optimal areal rainfall estimation using raingauges and satellite data, J. Hydrol., 222, 93-108, 1999.

Jobard, I., Chopin, F., Berges, J., Ali, A., Lebel, T., and Desbois, M.: Presentation of the EPSAT-SG method and comparison with other satellite precipitation estimations in the frame of PrecipAMMA, in: EGU conference proceedings, Vienna, 15-20 April 2007, 2007. 
Key, J. and Intrieri, J.: Cloud Particle Phase Determination with the AVHRR, J. Appl. Meteorol., 39, 1797-1804, 2000.

Kidd, C.: Satellite rainfall climatology: A review, Int. J. Climatol., 21, 1041-1066, 2001.

Laws, K., Janowiak, J., and Huffman, G.: Verification of rainfall estimates over Africa using RFE, NASA MPA-RT and CMORPH., in: American Meteorological Society Annual Conference, Seattle, Washington, 11-15 January 2004, 2004.

Lensky, I. and Rosenfeld, D.: A night-rain delineation algorithm for infrared satellite data based on microphysical considerations., J. Appl. Meteorol., 42, 1218-1226, 2003.

Lovejoy, S. and Austin, G.: The delineation of rain areas from visible and IR satellite data for GATE and mid-latitudes, Atmos.Ocean, 17, 77-92, 1979.

Morgan, J., ed.: Meteosat Second Generation. In Orbit - In Use, EUMETSAT, Darmstad, Germany, 2005.
Nzeukou, A., Sauvageot, H., Ochou, A., and Kebe, C.: Raindrop Size Distribution and Radar Parameters at Cape Verde, J. Appl. Meteorol., 43, 90-105, 2004.

Ochou, A., Nzeukou, A., and Sauvageot, H.: Parametrization of drop size distribution with rain rate, Atmos. Res., 84, 58-66, 2007.

Thies, B., Nauss, T., and Bendix, J.: Discriminating raining from non-raining clouds at mid-latitudes using meteosat second generation daytime data, Atmos. Chem. Phys., 8, 2341-2349, 2008, http://www.atmos-chem-phys.net/8/2341/2008/.

Thies, B., Nauss, T., and Bendix, J.: Discriminating raining from non-raining cloud area at mid-latitudes using MSG SEVIRI night-time data, Meteorol. Appl., 15, 219-230, 2008b.

Todd, M., Kidd, C., Kniveton, D., and Bellerby, T.: A combined satellite infrared and passive microwave technique for estimation of small-scale rainfall, J. Atmos. Oceanic Technol., 18, 742-755, 2001. 